\title{
CONTINUITY PROPERTIES OF THE BALL HULL MAPPING
}

\author{
J. P. MORENO AND R. SCHNEIDER
}

\begin{abstract}
The ball hull mapping $\beta$ associates with each closed bounded convex set $K$ in a Banach space its ball hull $\beta(K)$, defined as the intersection of all closed balls containing $K$. We are concerned in this paper with continuity and Lipschitz continuity (with respect to the Hausdorff metric) of the ball hull mapping. It is proved that $\beta$ is a Lipschitz map in finite dimensional polyhedral spaces. Both properties, finite dimension and polyhedral norm, are necessary for this result. Characterizing the ball hull mapping by means of H-convexity we show, with the help of a remarkable example from combinatorial geometry, that there exist norms with noncontinuous $\beta$ map, even in finite dimensional spaces. Using this surprising result, we then show that there are infinite dimensional polyhedral spaces (in the usual sense of Klee) for which the map $\beta$ is not continuous. A property known as ball stability implies that $\beta$ has Lipschitz constant one. We prove that every Banach space of dimension greater than two can be renormed so that there is an intersection of closed balls for which none of its parallel bodies is an intersection of closed balls, thus lacking ball stability.
\end{abstract}

\section{INTRODUCTION}

The family $\mathcal{M}$ of all intersections of closed balls in a real Banach space $X$ appears in several questions related to convex geometry, fixed point theory and geometry of Banach spaces. In the latter context, Mazur initiated some 75 years ago the study of the spaces satisfying $\mathcal{M}=\mathcal{H}$, where $\mathcal{H}$ denotes the family of all closed bounded convex sets in $X$, a property that now is known as the Mazur intersection property. It received later renewed attention by Phelps, Giles and Bor-Luh Lin, among many other authors. However, some of the basic properties of $\mathcal{M}$ when $\mathcal{M} \neq \mathcal{H}$ were still unknown. For instance, when $\mathcal{H}$ is equipped with the usual Hausdorff metric, is it true or false that $\mathcal{M}$ is (topologically) closed? This question (answered in the negative in Section 3) is related to the property of $\mathcal{M}$ being closed under Minkowski sums, and to another stability property concerning parallel bodies. We say that $\mathcal{M}$ is (i) stable if $\overline{C+D} \in \mathcal{M}$ whenever $C, D \in \mathcal{M}$; (ii) ball stable if $\overline{C+B} \in \mathcal{M}$ for every $C \in \mathcal{M}$, where $B$ is the (closed) unit ball. While it is obvious that $\mathcal{M}$ stable implies $\mathcal{M}$ ball stable, the converse is false [6]. It was shown in [11] that the ball stability of $\mathcal{M}$ implies that the ball hull mapping $\beta$ is Lipschitz. The

2000 Mathematics Subject Classification: 46B20, 52A21

The first autor was partially supported by the DGICYT project BFM 2003-06420. The second author is partially supported by the European Network PHD, FP6 Marie Curie Actions, RTN, Contract MCRN511953.

Key words and phrases. Intersections of balls, ball hull mapping, polyhedral norms, semi-denting point, H-convexity. 
ball hull mapping associates with every set $K \in \mathcal{H}$ its ball hull $\beta(K)$, the intersection of all closed balls containing $K$. It is not difficult to prove that $\mathcal{M}$ is closed if $\beta$ is continuous.

We will show in Section 2 that every Banach space of dimension greater than two can be renormed to lack ball stability. The class of norms used in the proof will provide a counterexample to show that $\mathcal{M}$ need not be ball stable when $\beta$ is Lipschitz. A main tool in this and the subsequent sections is the notion of semi-denting point introduced by Chen and Lin in [3].

In Section 3 we characterize the ball convex hull through H-convexity, a notion introduced by Boltyanski in [1]. We use this to show, by employing a remarkable example in [2], that there exist noncontinuous $\beta$ maps, even in finite dimensional spaces. In Section 4 it is proved that this cannot happen when the norm is polyhedral, since in this case, $\beta$ is always Lipschitz. We show that the condition of finite dimension is essential, by exhibiting a polyhedral norm in $c_{0}$ whose ball hull mapping is not continuous. When the space is not finite dimensional, we understand polyhedral always in the sense of Klee [9]. The following diagram summarizes some results of the present paper (together with known or obvious results):

$$
\begin{gathered}
\mathcal{M} \text { is ball stable } \\
\Downarrow \text { 丹 } \\
\beta \text { is Lipschitz } \\
\Downarrow \quad \Uparrow ? \\
X \text { polyhedral } \nRightarrow \quad \text { is continuous } \\
\Downarrow \text { polyhedral, } \operatorname{dim} X<\infty \\
\mathcal{M} \text { is closed }
\end{gathered}
$$

It also points out that there are questions concerning the continuity of ball hull mappings which are still unsolved. For instance, we have no example of a continuous $\beta$ map which is not Lipschitz. It has been observed in [13] that the Lipschitz property of $\beta$ with Lipschitz constant 1 implies ball stability.

\section{SPACES LACKING BALL STABILITY}

Among the several classes of extreme points that can be found in the literature, the notion of semi-denting point, introduced by Chen and Lin in [3], is surely the most suitable for studying convex sets which are intersections of balls (every ball considered in this paper is closed). Recall that an element $f$ of the unit sphere $S^{*}$ of a dual Banach space is called a semi-denting point of the dual unit ball $B^{*}$ if for every $\varepsilon>0$ there exists a weak* slice $S$ such that $\operatorname{diam}(f \cup S)<\varepsilon$. If we ask additionally that $f \in S$, we recapture the notion of weak* denting point. The utility of semi-denting points can be illustrated by a result that will be used later in the proof of Theorem 2.2: if $f \in S^{*}$ and $C \in \mathcal{M}$ has a facet defined by a supporting hyperplane $H$ parallel to $f^{-1}(0)$ (here facet means nonempty relative interior in $H$ ), then $f$ is a semi-denting point of $B^{*}$. More precisely, 
we have the following lemma, whose proof is similar to the proof of Proposition 2.3 in [7] and, therefore, is omitted.

Lemma 2.1. If $f \in S^{*}, C \in \mathcal{M}, \alpha=\sup f(C)$ and $f^{-1}(\alpha) \cap C$ has nonempty (relative) interior in $f^{-1}(\alpha)$, then $f$ is a semi-denting point of $B^{*}$.

In every two dimensional Banach space, the elements of $\mathcal{M}$ satisfy a strong separation property which, in particular, implies that $\mathcal{M}$ is stable, hence ball stable [6]. In higher dimensions, the situation changes drastically, as the following theorem shows. Here, a parallel body of the set $C$ is any set of the form $\overline{C+\lambda B}$ where $B$ is the unit ball and $\lambda \geq 0$.

Theorem 2.2. A Banach space $X$ with $\operatorname{dim} X>2$ admits an equivalent norm for which there is an intersection of closed balls whose parallel bodies are not intersections of closed balls.

Proof. Assume that $\operatorname{dim} X>2$. Choose $f \in S^{*}$, consider the hyperplane $Y=f^{-1}(0)$, a norm-one vector $y \in Y$ and a functional $g \in S^{*}$ supporting $B$ at $y$. Since $\operatorname{dim} X>2$, there exists $z \in g^{-1}(0) \cap Y$ satisfying $\|z\|=1$. We can pick $x \in B$ with $f(x)=3 / 4$. We may assume that $-1<g(x)=\beta<1$. Finally, we can find $h \in S^{*}$ such that $h(x)=0$ and $h(z) \neq 0$. We can decompose $Y=\operatorname{lin}(z) \oplus H$, where $H=h^{-1}(0) \cap Y$. Consider $D=H \cap|\beta / 2| B$ and denote by $[y-z, y+z]$ the segment with end-points $y-z$ and $y+z$, respectively. The set

$$
B^{\prime}=\overline{\operatorname{conv}}([y-z, y+z] \cup[-y-z,-y+z] \cup(x+D) \cup(-x+D) \cup(1 / 2) B)
$$

is the unit ball of a norm $|\cdot|$ satisfying $(1 / 2)\|x\| \leq|x| \leq 2\|x\|$, hence equivalent to $\|\cdot\|$. Consider the set $C=B^{\prime} \cap\left(2 y+B^{\prime}\right) \in \mathcal{M}$. Our choice of $D$ and $B^{\prime}$ ensures that $C \subset g^{-1}(1)$ and, therefore,

$$
C=[y-z, y+z]
$$

What about $\overline{C+\lambda B^{\prime}}$ ? In this particular case, $\overline{C+\lambda B^{\prime}}=C+\lambda B^{\prime}$. Observe also that $\sup f\left(C+\lambda B^{\prime}\right)=\lambda \sup f\left(B^{\prime}\right)=3 \lambda / 4$. We claim that $f^{-1}(3 \lambda / 4) \cap\left(C+\lambda B^{\prime}\right)$ has nonempty relative interior in $f^{-1}(3 \lambda / 4)$. Indeed, $Y$ is isomorphic to $\operatorname{lin}(z) \oplus_{\infty} H$ and, therefore, the set $[-z, z]+\lambda(H \cap|\beta / 2| B)=[-z, z]+\lambda D$ has nonempty interior in $Y$. Now, observe that

$$
\begin{aligned}
f^{-1}(3 \lambda / 4) \cap\left(C+\lambda B^{\prime}\right) & =f^{-1}(3 \lambda / 4) \cap\left([y-z, y+z]+\lambda B^{\prime}\right) \\
& \supset[y-z, y+z]+\lambda x+\lambda D \\
& =(y+\lambda x)+([-z, z]+\lambda D),
\end{aligned}
$$

which proves the claim. Suppose that $C+\lambda B^{\prime} \in \mathcal{M}$. According to Lemma 2.1, $\varphi=f /|f|^{*}$ should be a semi-denting point of the new dual ball $\left(B^{\prime}\right)^{*}$. We will show that this is not the case, obtaining a contradiction. The idea is proving that, close enough to $\varphi$, every functional in the dual unit sphere $\left(S^{\prime}\right)^{*}$ is the center of a segment of $\|\cdot\|^{*}$-length $1 / 6$ lying 
in $\left(S^{\prime}\right)^{*}$. Hence, every weak* slice of $\left(B^{\prime}\right)^{*}$ containing points which are close to $\varphi$ has $\|\cdot\|^{*}$-diameter at least $1 / 12$.

The work now consists essentially in doing some calculations with dual norms. It is clear that $\sup f\left(B^{\prime}\right)=\sup f(x+D)=f(x)=3 / 4$, so $\varphi=(4 / 3) f$ and thus $\|\varphi\|^{*}=4 / 3$. As a first step, we show that $|\phi|^{*}=1$ and $\|\varphi-\phi\|^{*}<1 / 3$ imply, as in the case of $\varphi$, that $\sup \phi(x+D)=1$. Indeed,

$$
\begin{aligned}
\sup \phi([y-z, y+z] \cup[-y-z,-y+z]) & \leq \sup \phi(2 B \cap Y) \\
& \leq \sup \varphi(2 B \cap Y)+2\|\varphi-\phi\|^{*} \leq \frac{2}{3}
\end{aligned}
$$

and, analogously,

$$
\sup \phi\left(\frac{1}{2} B\right) \leq \frac{1}{2}(\sup \varphi(B)+\sup (\phi-\varphi)(B)) \leq \frac{1}{2}\left(\frac{4}{3}+\frac{1}{3}\right)=\frac{5}{6} .
$$

Now we claim that $|\phi \pm h / 6|^{*}=1$ whenever $|\phi|^{*}=1$ and $\|\varphi-\phi\|^{*}<1 / 3$. Indeed,

$$
\begin{aligned}
\sup (\phi \pm h / 6)\left(B^{\prime}\right) & \geq \sup \phi(x+D)-\sup (h / 6)(x+D) \\
& =\sup \phi(x+D)=1 .
\end{aligned}
$$

To prove that $\sup (\phi \pm h / 6)\left(B^{\prime}\right) \leq 1$, we first notice, on the one hand, that $\sup (\phi \pm$ $h / 6)(x+D)=\sup \phi(x+D)=1$ as we observed above, and $\sup (\phi \pm h / 6)(-x+D)=$ $\sup \phi(-x+D) \leq \sup \phi\left(B^{\prime}\right)=1$. On the other hand, by using (2) we get

$$
\sup (\phi \pm h / 6)\left(\frac{1}{2} B\right) \leq \sup \phi\left(\frac{1}{2} B\right)+\frac{1}{12}\|h\|^{*} \leq \frac{11}{12} .
$$

Finally, according to (1),

$$
\sup (\phi \pm h / 6)([y-z, y+z] \cup[-y+z,-y-z]) \leq \frac{2}{3}+\frac{1}{6} \sup h(2 B \cap Y) \leq 1 .
$$

Notice that ball stability has a trivial meaning in spaces with the Mazur intersection property since, in these spaces, every closed bounded convex set is an intersection of closed balls. When the space lacks the Mazur intersection property then there are always functionals which are not semi-denting [3].

The ball hull mapping associates with every closed bounded convex set $K$ its ball hull $\beta_{\|\cdot\|}(K)$ (or simply $\beta(K)$ when it causes no confusion), namely the intersection of all closed balls containing $K$. It is known [11] that $\beta$ is Lipschitz when $\mathcal{M}$ is ball stable. The Lipschitz property here refers to the usual Hausdorff distance:

$$
\operatorname{dist}_{\|\cdot\|}(C, D)=\inf \left\{\varepsilon>0: C \subset D+\varepsilon B_{\|\cdot\|} \text { and } D \subset C+\varepsilon B_{\|\cdot\|}\right\} .
$$


We will use the construction employed in Theorem 2.2 to prove that the converse is false.

Proposition 2.3. $\mathcal{M}$ need not be ball stable when $\beta$ is Lipschitz.

Proof. Denote by $\|\cdot\|_{1}$ the usual $\ell_{1}$ norm in $\mathbb{R}^{3}$ and let $e_{1}, e_{2}, e_{3}$ be the standard orthonormal basis of $\mathbb{R}^{3}$. Consider the point $u=\frac{1}{2} e_{1}+\frac{1}{2} e_{2}$, the segment $[-u, u]$ and the new norm $|\cdot|$ whose unit ball is the set $B_{|\cdot|}=[-u, u]+B_{\|\cdot\|_{1}}$. By using the same arguments as in Theorem 2.2, we get that the family $\mathcal{M}_{|\cdot|}$ associated with $|\cdot|$ is not ball stable. However, we claim that the $|\cdot|$-ball hull mapping (that is, the ball hull mapping associated with $|\cdot|)$ is Lipschitz with exact constant 2. In order to prove the claim it is important to notice first that

$$
\mathcal{M}_{|\cdot|}=\mathcal{M}_{\|\cdot\|_{1}}
$$

The inclusion $\mathcal{M}_{|\cdot|} \supset \mathcal{M}_{\|\cdot\|_{1}}$ is easy because $B_{\|\cdot\|_{1}}$ is an intersection of two $|\cdot|$-balls. The reverse inclusion holds because $B_{|\cdot|} \in \mathcal{M}_{\|\cdot\|_{1}}$. This fact can be intuitively explained as follows: $B_{|\cdot|}$ is the intersection of eight halfspaces defined by the four functionals $f_{1}\left(x_{1}, x_{2}, x_{3}\right)=x_{1}+x_{2}+x_{3}, \quad f_{2}\left(x_{1}, x_{2}, x_{3}\right)=x_{1}+x_{2}-x_{3}, \quad f_{3}\left(x_{1}, x_{2}, x_{3}\right)=x_{1}-$ $x_{2}-x_{3}, \quad f_{4}\left(x_{1}, x_{2}, x_{3}\right)=x_{1}-x_{2}+x_{3}$ which are used to define $B_{\|\cdot\|_{1}}$. Then, eight suitable homothetic copies of $B_{\|\cdot\|_{1}}$ can play the role of these eight halfspaces. This shows immediately that $B_{|\cdot|} \in \mathcal{M}_{\|\cdot\|_{1}}$ Consider now two bounded sets $C, D$ satisfying $\operatorname{dist}_{|\cdot|}(C, D)=\varepsilon$ and denote by $\beta_{|\cdot|}(C), \beta_{|\cdot|}(D)$ the corresponding $|\cdot|$-ball hulls. Then, since $B_{|\cdot|} \subset 2 B_{\|\cdot\|_{1}}$, we have

$$
C \subset D+\varepsilon B_{|\cdot|} \subset D+2 \varepsilon B_{\|\cdot\|_{1}} \subset \beta_{|\cdot|}(D)+2 \varepsilon B_{\|\cdot\|_{1}} .
$$

The important point here is that $\mathcal{M}_{\|\cdot\|_{1}}$ is $\|\cdot\|_{1}$-ball stable [6], hence

$$
C \subset \beta_{|\cdot|}(D)+2 \varepsilon B_{\|\cdot\|_{1}} \in \mathcal{M}_{\|\cdot\|_{1}}=\mathcal{M}_{|\cdot|}
$$

and therefore

$$
\beta_{|\cdot|}(C) \subset \beta_{|\cdot|}(D)+2 \varepsilon B_{\|\cdot\|_{1}}
$$

Using that $B_{\|\cdot\|_{1}} \subset B_{|\cdot|}$, we conclude that $\beta_{|\cdot|}(C) \subset \beta_{|\cdot|}(D)+2 \varepsilon B_{|\cdot| \cdot}$. A symmetric argument yields $\beta_{|\cdot|}(D) \subset \beta_{|\cdot|}(C)+2 \varepsilon B_{|\cdot|}$, hence dist||| $\left(\beta_{|\cdot|}(C), \beta_{|\cdot|}(D)\right) \leq 2 \varepsilon$ and so the $|\cdot|$-ball hull map is Lipschitz with constant 2. To see that the constant 2 is sharp, consider the two segments $C=\left[-e_{2}, e_{2}\right]$ and $D=\left[-\frac{1}{2} e_{1}+\frac{1}{2} e_{2}+e_{3}, \frac{1}{2} e_{1}-\frac{1}{2} e_{2}+e_{3}\right]$. Then $\operatorname{dist}_{|\cdot|}(C, D)=1$ while $\operatorname{dist}_{|\cdot|}(\beta(C), \beta(D))=2$.

Though it is clear from the above result that a Lipschitz $\beta$ mapping does not imply the ball stability of $\mathcal{M}$, we don't know whether such a result is true if we ask, in addition, that the ball hull mapping be Lipschitz with constant 1 . Besides, we have no example of a continuous ball hull mapping which is not Lipschitz. 


\section{The BALl hull MAPping AND H-CONVEXITY}

The aim of this section is to prove that, quite surprisingly, there are norms for which the ball hull mapping is not continuous. The key to prove this result is to establish a suitable connection between the ball hull mapping and $H$-convexity, a notion introduced by Boltyanski in [1] in the context of finite dimensional euclidean spaces. It admits an easy generalization to arbitrary (even infinite dimensional) Banach spaces. The basic idea is the following. Let $H$ denote a subset of $X^{*} \backslash\{0\}$; the set $C \subset X$ is said to be $H$-convex if it can be represented as

$$
C=\bigcap_{f \in H} f^{-1}(-\infty, \sup f(C)]
$$

where $\sup f(C)$ can be $\infty$. $H$-convex sets are always convex and closed; however, they need not be bounded. The question now is to decide which properties of $H$ ensure the existence of an equivalent norm $|\cdot|$ such that $H$-convex sets coincide with the family $\mathcal{M}_{|\cdot|}$ of all intersections of $|\cdot|$-closed balls. Recall that a subset $F$ of the dual unit sphere $S^{*}$ is called $\lambda$-norming if $\|x\| \leq \lambda \sup _{f \in F} f(x)$ for every $x \in X$. We say that $F$ is norming if it is $\lambda$-norming for some $\lambda \geq 1$. Of course, $S^{*}$ is a trivial 1-norming set but, in general, norming sets need not be (norm) dense in $S^{*}$. Consider, for instance, $F=\{ \pm(1,0), \pm(0,1)\} \subset \ell_{1}^{2}$, the dual space of $\ell_{\infty}^{2}$. An element $f$ of the unit sphere $S^{*}$ of a dual Banach space is called a weak* denting point if for every $\varepsilon>0$ there exists a weak* slice $S$ of $B^{*}$ such that $\operatorname{diam} S<\varepsilon$ and $f \in S$. A subset $H \subset S^{*}$ is symmetric if $f \in H$ implies $-f \in H$.

Theorem 3.1. Let $H \subset S^{*}$ be a symmetric norming set of weak* denting points. There exists an equivalent norm $|\cdot|$ such that $\mathcal{M}_{|\cdot|}$ is precisely the set of $H$-convex subsets.

Proof. Consider the set

$$
D=\bigcap_{f \in H} f^{-1}(-\infty, 1]=\bigcap_{f \in H} f^{-1}[-1,1]
$$

which is closed, convex, centrally symmetric and contains $B$. Moreover, since $H$ is $\lambda$ norming for some $\lambda \geq 1$, we have $D \subset \lambda B$, hence $D$ is also bounded. In other words, $D$ is the unit ball of the equivalent norm

$$
|x|=\sup _{f \in H}|f(x)|
$$

which satisfies $|x| \leq\|x\| \leq \lambda|x|$. If $C \in \mathcal{M}_{|\cdot|}$, there is a collection of balls $\left\{D_{\alpha}=\right.$ $\left.\bigcap_{f \in H} f^{-1}\left[a_{\alpha}, b_{\alpha}\right]\right\}_{\alpha}$ such that $C=\bigcap_{\alpha} D_{\alpha}$. Consequently,

$$
C=\bigcap_{f \in H} f^{-1}\left[\sup _{\alpha}\left\{a_{\alpha}\right\}, \inf _{\alpha}\left\{b_{\alpha}\right\}\right]
$$


and therefore $C$ is $H$-convex. To prove the converse, let us first check that the functionals in $H$ are weak* denting points of the new dual unit ball $D^{*}$. Indeed, notice first that $|f|^{*}=1$ for every $f \in H$, since $\sup |f(D)| \leq 1$ by (4) and $\sup |f(D)| \geq 1$ because $B \subset D$. Now, given $f \in H$ and $\varepsilon>0$, there is a weak ${ }^{*}$ closed halfspace $W \in X^{*}$ containing $f$ and satisfying $\operatorname{diam}_{\|\cdot\|^{*}}\left(W \cap B^{*}\right)<\lambda^{-1} \varepsilon$. It is clear that $W \cap D^{*} \neq \emptyset$ because $f \in W \cap D^{*}$ (this is the reason why we are considering weak* denting points instead of semi-denting points). Now, since $D^{*} \subset B^{*}$ and $|g| \leq \lambda\|g\|$ for every $g \in X^{*}$, we have

$$
\operatorname{diam}_{|\cdot|^{*}}\left(W \cap D^{*}\right) \leq \operatorname{diam}_{|\cdot|^{*}}\left(W \cap B^{*}\right) \leq \lambda \operatorname{diam}_{\|\cdot\|^{*}}\left(W \cap B^{*}\right)<\varepsilon,
$$

which proves that $f$ is a weak ${ }^{*}$ denting point of $D^{*}$. In particular, $f$ is also a semi-denting point. Let us see that a set $C$ satisfying (3) is an intersection of balls, that is $C \in \mathcal{M}_{|\cdot|}$. To this end, consider $x \notin C$. There is an $f \in H$ such that $f(x) \notin(-\infty, \sup f(C)]$. The Chen-Lin characterization of semi-denting points [3] already used in the previous section implies the existence of a ball containing $C$ but missing $x$.

It is not difficult to prove that the continuity of the ball hull mapping implies that $\mathcal{M}$ is closed. Boltyanski, Martini and Soltan proved in Example 20.6 of [2] that, for $\mathbb{R}^{3}$ and a suitable choice of $H$, there is a sequence of $H$-convex sets converging to a set $M$ which is not $H$-convex. It is not difficult to check that this particular $H$ is a symmetric norming set of weak* denting points and thus satisfies the conditions of Theorem 3.1. Applying this theorem, we get a norm $|\cdot|$ for which $\mathcal{M}_{|\cdot|}$ is not closed, hence the $|\cdot|$-ball hull mapping is not continuous. Let us remark that $M$ has empty interior (it is a line segment in $\mathbb{R}^{3}$ ). This is necessarily so, due to the following fact.

Proposition 3.2. The ball hull mapping is always continuous at sets which have nonempty interior.

Proof. Consider a set $C \in \mathcal{H}$ such that int $C \neq \emptyset$ and a sequence $C_{n} \rightarrow C$ in $\mathcal{H}$. We may assume that $0 \in \operatorname{int} C$. For every $\lambda>1$ we consider the homothetic sets $\lambda C$ and $\lambda^{-1} C$, which satisfy $\lambda^{-1} C \subset C \subset \lambda C$. Now, every family $\left\{B_{i}\right\}$ of closed balls and every $\mu \in \mathbb{R}$ satisfy $\bigcap_{i} \mu B_{i}=\mu \bigcap_{i} B_{i}$. Therefore, $\beta(\lambda C)=\lambda \beta(C)$ and, similarly, $\beta\left(\lambda^{-1} C\right)=\lambda^{-1} \beta(C)$. This implies

$$
\lambda^{-1} \beta(C) \subset \beta(C) \subset \lambda \beta(C) .
$$

It is easily verified that, if $\|x\| \leq M$ for all $x \in C$ (and hence for all $x \in \beta(C)$ ), then we obtain, using the Hausdorff metric, that

$$
\operatorname{dist}\left(\lambda^{-1} \beta(C), \lambda \beta(C)\right) \leq \lambda\left(1-1 / \lambda^{2}\right) M
$$

Given $\varepsilon>0$, fix $\lambda>1$ such that $\lambda\left(1-1 / \lambda^{2}\right) M<\varepsilon$. Now, the set $\left\{D \in \mathcal{H}: \lambda^{-1} C \subset D \subset\right.$ $\lambda C$ is a neighborhood of $C$ and, consequently, $\lambda^{-1} C \subset C_{n} \subset \lambda C$ for all sufficiently large $n$ (say for $n \geq N)$, so $\lambda^{-1} \beta(C) \subset \beta\left(C_{n}\right) \subset \lambda \beta(C)$ for $n \geq N$, and hence $\operatorname{dist}\left(\beta\left(C_{n}\right), \beta(C)\right)<$ $\varepsilon$ for $n \geq N$. 
The question of whether $C \in \mathcal{M}$ whenever $\overline{C+\lambda B} \in \mathcal{M}$ for every $\lambda>0$ can be easily answered in the affirmative. Indeed, if $x \notin C$, there is $\lambda>0$ such that $x \notin \overline{C+\lambda B}$. Since $\overline{C+\lambda B} \in \mathcal{M}$, there is a closed ball $D$ satisfying $C \subset \overline{C+\lambda B} \subset D$ and $x \notin D$. On the other hand, if we define $C \sim \lambda B=\{x \in C: x+\lambda B \subset C\}$, it is natural to ask also whether $C \in \mathcal{M}$ whenever $\emptyset \neq C \sim \lambda B \in \mathcal{M}$ for every sufficiently small $\lambda>0$. To prove that this is indeed the case, first notice that $\lim _{\lambda \rightarrow 0} \operatorname{dist}(C, C \sim \lambda B)=0$ (since int $C \neq \emptyset$ and $C$ is convex) and then apply Proposition 3.2.

Theorem 3.1 answers the question of finding, given a set $H \subset S^{*}$, an equivalent norm | | such that the family of $H$-convex sets coincides with $\mathcal{M}_{|\cdot|}$. Conversely, if we fix the norm $\|\cdot\|$, is there a nontrivial $H \subset S^{*}$ with the property that $\mathcal{M}_{\|\cdot\|}$ is precisely the family of $H$-convex sets? The natural candidate is $H=S D^{*}$, the set of semi-denting points. By using similar arguments as in the proof of the above theorem, one gets the following representation result. If $S D^{*}$ is a 1-norming set, then $C \in \mathcal{M}$ if and only if

$$
\begin{aligned}
C & =\bigcap_{f \in S D^{*}} f^{-1}[\inf f(C), \sup f(C)] \\
& =\bigcap_{f \in S D^{*}} f^{-1}(-\infty, \sup f(C)]=\bigcap_{f \in S D^{*}} f^{-1}[\inf f(C), \infty),
\end{aligned}
$$

where the last two equalities are due to the fact that $f \in S D^{*}$ if and only if $-f \in S D^{*}$. In other words, if $S D^{*}$ is 1-norming, then $\mathcal{M}$ is the family of $H$-convex sets, for $H=S D^{*}$. If $\left\{x_{i}\right\}_{i \in I}$ is any dense set of the unit sphere $S$ and $\left\{f_{i}\right\} \subset S^{*}$ satisfies $f_{i}\left(x_{i}\right)=1$ for every $i \in I$, then $\left\{f_{i}\right\}$ is a norming set. When $X$ is an Asplund space, there is a dense set of points $\left\{x_{i}\right\}_{i \in I} \subset S$ and corresponding support functionals $\left\{f_{i}\right\}_{i \in I} \subset S^{*}$ such that the $f_{i}$ are weak* strongly exposed points of $B^{*}$ (thus obviously they are also semi-denting). Points with the above property are usually called regular or smooth points. Consequently, if $X$ is Asplund (in particular, if $X$ has finite dimension), then $C \in \mathcal{M}$ if and only it can be represented as in (6), replacing $S D^{*}$ by $S E^{*}$, the family of weak ${ }^{*}$ strongly exposed points of $B^{*}$. The representation given in (6) does not mean that, in general, the functionals of $S D^{*}$ support every boundary point of $C$. However, this is the case when the space has finite dimension, as proved in the following corollary.

Corollary 3.3. Let $X$ be a finite dimensional Banach space. If $C$ is an intersection of closed balls and $x \in C$ is a boundary point, there is $f \in S D^{*}$ supporting $C$ at $x$.

Proof. Choose a sequence $\left\{x_{n}\right\}_{n} \subset X \backslash C$ converging to $x$. Since $C$ satisfies (6), for every $n \in \mathbb{N}$ we can choose $f_{n} \in S D^{*}$ such that

$$
f_{n}\left(x_{n}\right) \notin\left[\inf f_{n}(C), \sup f_{n}(C)\right]
$$

while, obviously, $f_{n}(x) \in\left[\inf f_{n}(C), \sup f_{n}(C)\right]$. Since $S^{*}$ is compact and $S D^{*}$ is closed (a result whose proof can be deduced directly from the definition of a semi-denting point), there is a subsequence $\left\{f_{n_{k}}\right\}_{k} \subset\left\{f_{n}\right\}_{n}$ converging to $f_{0} \in S D^{*}$. Notice that either $f_{0}(x)=\inf f_{0}(C)$ or $f_{0}(x)=\sup f_{0}(C)$. Otherwise, if $f_{0}(x) \in\left(\inf f_{0}(C)\right.$, sup $\left.f_{0}(C)\right)$, then 
$f_{0}\left(x_{n}\right) \in\left(\inf f_{0}(C), \sup f_{0}(C)\right)$ for $n$ big enough, hence $f_{n}\left(x_{n}\right) \in\left(\inf f_{n}(C), \sup f_{n}(C)\right)$ for some $n$, a contradiction.

Remark: It can be useful to observe that in a finite dimensional normed space the semidenting points are precisely the limits of extreme points. Indeed, in these spaces, the notions of extreme, denting and weak* denting point coincide. Hence, every extreme point of $B^{*}$ is semi-denting. On the other hand, by the Minkowski theorem (see [12], Lemma 1.4.5), we have $B^{*}=\operatorname{conv}\left(\operatorname{ext}\left(B^{*}\right)\right)$ and then every nonempty slice of $C$ contains an extreme point. Together with the definition of semi-denting point, this proves the assertion.

Finally, in spaces with infinite dimension, extreme points need not be semi-denting. Consider, for instance, the usual unit ball of $\ell_{\infty}$. It contains no semi-denting points, since every weak* ${ }^{*}$ slice has diameter 2 , but it certainly contains extreme points.

\section{BALl HULL MAPPINGS OF POLYHEDRAL NORMS}

A polytope is the convex hull of finitely many points. The theory of polytopes plays an important role in convex geometry and other branches of mathematics (for introductions, see Grünbaum [8] and Ziegler [14]). When the unit ball of a finite dimensional Banach space is a polytope, we say that both the space and the norm are polyhedral. One of the several attempts to define infinite dimensional polyhedral spaces (and probably the one most accepted nowadays) is due to V. Klee [9]: a space is polyhedral if each of the finite dimensional sections of its unit ball is a polytope. Analogously, its norm is usually called a polyhedral norm. For classical and recent results on this and other versions on infinite-dimensional polyhedrality, the reader is referred to [5].

A subset $F \subset S^{*}$ is called a boundary if for each $x \in X$ there exists $f \in F$ such that $f(x)=\|x\|$. Finite dimensional polyhedral spaces have finite boundaries and every polyhedral Banach space admits a boundary $\Omega=\left\{f_{i}\right\}_{i \in I} \subset S^{*}$ with the property that $f_{i}^{-1}(1) \cap S$ has nonempty relative interior in $f_{i}^{-1}(1)$, for every $i \in I$ [4]. It can be used to represent the elements of $\mathcal{M}$ as follows. Since $f_{i}^{-1}(1) \cap S$ has nonempty relative interior in $f_{i}^{-1}(1)$, the functional $f_{i}$ is a weak* strongly exposed point of $B^{*}$, thus a semi-denting point. As a consequence, if $C \in \mathcal{M}$, then

$$
C=\bigcap_{i \in I} f_{i}^{-1}\left(-\infty, \sup f_{i}(C)\right] .
$$

Theorem 4.1. In a finite dimensional polyhedral space, the ball hull mapping is Lipschitz continuous.

Proof. Let $X$ be a finite dimensional polyhedral space. Consider the finite boundary $\Omega=\left\{f_{i}\right\}_{i \in I}$ described before. We will show that there is a constant $c>0$ such that, for 
$C \in \mathcal{M}$ and $x \in X \backslash C$,

$$
\operatorname{dist}(x, C) \leq c \sup _{i}\left\{f_{i}(x)-\sup f_{i}(C)\right\}
$$

Before proving (10), let us see why this inequality is the key to proving the theorem. Consider a closed bounded convex set $K \subset X$ and $\lambda>0$. We claim that

$$
\beta(K+\lambda B) \subset \beta(K)+c \lambda B .
$$

For the proof, let $x \in X$ be such that $x \notin \beta(K)+c \lambda B$, then $\operatorname{dist}(x, \beta(K))>c \lambda$ and hence, according to (10), we have

$$
\begin{aligned}
c \lambda & <c \sup _{i}\left\{f_{i}(x)-\sup f_{i}(\beta(K))\right\} \\
& =c \sup _{i}\left\{f_{i}(x)-\sup f_{i}(K)\right\}
\end{aligned}
$$

where (9) was used. This implies the existence of $j \in I$ satisfying

$$
\lambda<f_{j}(x)-\sup f_{j}(K) .
$$

This inequality, in turn, yields that $x \notin \beta(K+\lambda B)$, since

$$
\beta(K+\lambda B) \subset f_{j}^{-1}\left(-\infty, \sup f_{j}(K)+\lambda\right] .
$$

To prove the theorem, let $K_{1}, K_{2}$ be closed bounded convex sets with $\operatorname{dist}\left(K_{1}, K_{2}\right)=\delta$. Then, $K_{1} \subset K_{2}+\delta B$ and so

$$
\beta\left(K_{1}\right) \subset \beta\left(K_{2}+\delta B\right) \subset \beta\left(K_{2}\right)+c \delta B
$$

which, together with the analogous expression obtained by interchanging $K_{1}$ and $K_{2}$, allows us to conclude that

$$
\operatorname{dist}\left(\beta\left(K_{1}\right), \beta\left(K_{2}\right)\right) \leq c \operatorname{dist}\left(K_{1}, K_{2}\right) .
$$

It remains to prove (10), with suitable $c$. For that, we choose a real number $c>0$ such that for any $k$ linearly independent functionals $f_{1}, \ldots, f_{k} \in \Omega$ and for $\alpha_{1}, \ldots, \alpha_{k} \in \mathbb{R}$ we have

$$
\left|\alpha_{1}\right|+\cdots+\left|\alpha_{k}\right| \leq c\left\|\alpha_{1} f_{1}+\cdots+\alpha_{k} f_{k}\right\|^{*}
$$

The existence of such a constant follows by a routine argument, using homogeneity, continuity, compactness, and the finiteness of $\Omega$. Now let $C \in \mathcal{M}$ and $x \in X \backslash C$ be given. Define $\varepsilon=\operatorname{dist}(x, C)$ and let $y$ be a point in $C$ with $\|x-y\|=\varepsilon$. The compact convex sets $\varepsilon B+x$ and $C$ have the point $y$ in common, but no relatively interior points, hence they can be separated by a hyperplane $H$ containing $y$. Thus, there exists $g \in X^{*}$ and $\alpha \in \mathbb{R}$ with $H=g^{-1}(\alpha), g(x)>\alpha, \sup g(C)=\alpha$, and $g(x-y)=\|g\|^{*}\|x-y\|=\varepsilon\|g\|^{*}$. 
The hyperplane $H$ supports $C$, and $F=H \cap C$ is a face of $C$. Let $\Omega_{F}=\left\{f_{1}, \ldots, f_{m}\right\}$ be the set of functionals $f \in \Omega$ for which

$$
F \subset f^{-1}(\sup f(C))
$$

that is, the supporting hyperplane of $C$ determined by $f$ contains $F$. Clearly, $\Omega_{F}$ is not empty. We assert that

$$
g \in \operatorname{pos}\left\{f_{1}, \ldots, f_{m}\right\}
$$

where $\operatorname{pos} A$ denotes the positive hull of $A$, i.e., the set of all linear combinations of elements from $A$ with nonnegative coefficients. Suppose, this were false. Then $g$ and the convex cone $\operatorname{pos}\left\{f_{1}, \ldots, f_{m}\right\}$ can be separated, that is, there exists a vector $w \in X$ such that $g(w)>0$ and $f_{i}(w) \leq 0$ for $i=1, \ldots, m$. Choose $z \in \operatorname{relint} F$, the relative interior of $F$. Then we have $g(z)=\sup g(C)$ and also, in virtue of $(13), f_{i}(z)=\sup f_{i}(C)$ for $i=1, \ldots, m$, while $f(z)<\sup f(C)$ for $f \in \Omega \backslash \Omega_{F}$ (otherwise, $f(z)=\sup f(C)$, and since $z \in \operatorname{relint} F$, this implies $F \subset f^{-1}(\sup f(C))$, a contradiction). We can choose $\lambda>0$ so small that

$$
f(z+\lambda w)<\sup f(C)
$$

for $f \in \Omega \backslash \Omega_{F}$. Since $f_{i}(z+\lambda w) \leq \sup f_{i}(C)$ for $i=1, \ldots, m$, we have $z+\lambda w \in$ $\bigcap_{f \in \Omega} f^{-1}(-\infty, \sup f(C)]=C$. On the other hand, $g(z+\lambda w)>\sup g(C)$, a contradiction. Thus (14) holds.

By (14), there is a representation $g=\sum_{i=1}^{m} \alpha_{i} f_{i}$ with $\alpha_{i} \geq 0$. By the analogue of Carathéodory's theorem for positive combinations, $g$ is a positive combination of linearly independent functionals among $f_{1}, \ldots, f_{m}$, say $g=\sum_{i=1}^{k} \alpha_{i} f_{i}$ with $\alpha_{i}>0$. If we would have $f_{i}(x-y)<c^{-1} \epsilon$ for $i=1, \ldots, k$, then, by (12),

$$
\varepsilon\|g\|^{*}=g(x-y)=\sum_{i=1}^{k} \alpha_{i} f_{i}(x-y)<c^{-1} \varepsilon \sum_{i=1}^{k} \alpha_{i} \leq c^{-1} \varepsilon c\|g\|^{*},
$$

a contradiction. Hence, there is $j \in\{1, \ldots, k\}$ with $f_{j}(x-y) \geq c^{-1} \varepsilon$, that is,

$$
\operatorname{dist}(x, C)=\varepsilon \leq c f_{j}(x-y)=c\left(f_{j}(x)-\sup f_{j}(C)\right),
$$

which proves (10) and thus the theorem.

We finish this section by showing that Theorem 4.1 is best possible, under two aspects: for a finite dimensional space, there is no upper bound for the Lipschitz constants of the ball hull mappings of polyhedral norms, and in an infinite dimensional polyhedral space, the ball hull mapping even need not be continuous.

Proposition 4.2. For every $n \in \mathbb{N}$, there is a polyhedral norm on $\mathbb{R}^{3}$ such that the corresponding ball hull map has (minimal) Lipschitz constant greater than $n$.

Proof. The intuitive idea is the following: if we choose a norm $\|\cdot\|$ in $\mathbb{R}^{3}$ whose associated ball hull map $\beta$ is not continuous, and if we approximate $\|\cdot\|$ by polyhedral norms $\|\cdot\|_{k}$, 
then the corresponding ball hull maps $\beta_{k}$ must have large minimal Lipschitz constant. A norm $\|\cdot\|$ with non-continuous $\beta$ map was obtained in Section 3, on the basis of Example 20.6 of [2]. We modify this example to construct now the approximating polyhedral norms.

We denote by $\langle\cdot, \cdot\rangle$ and $\left(e_{1}, e_{2}, e_{3}\right)$ the standard scalar product and orthonormal basis of $\mathbb{R}^{3}$, respectively. With every $u \in \mathbb{R}^{3}$, we associate the linear functional $u^{*}$ with $u^{*}(x)=$ $\langle u, x\rangle$ for $x \in \mathbb{R}^{3}$. Consider the segment $M=\left[-e_{2}, e_{2}\right]$ and the sequence of segments $M_{k}=\left[-e_{2}+\frac{1}{k} e_{3}, e_{2}-\frac{1}{k} e_{3}\right], k \in \mathbb{N}$. Define the vectors $f_{k}^{(+)}$and $f_{k}^{(-)}$as in [2], consider the set

$$
\Omega_{k}=\left\{ \pm f_{k}^{(+)}, \pm f_{k}^{(-)},\left( \pm e_{1} \pm e_{2}\right) / \sqrt{2}, \pm e_{3}\right\}
$$

and define

$$
B_{k}=\bigcap_{f \in \Omega_{k}^{*}} f^{-1}(-\infty, \sup f(D)),
$$

where $D$ is the euclidean unit ball. Then $B_{k}$ is the unit ball of a polyhedral norm $\|\cdot\|_{k}$. Note that $H_{k}=\Omega_{k}^{*}$ is a boundary of this norm. Let $\operatorname{dist}_{k}$ and $\beta_{k}$ denote the Hausdorff metric and ball hull mapping, respectively, of the norm $\|\cdot\|_{k}$. We have shown in Section 3 that the $\Omega_{k}^{*}$-convex hull map coincides with $\beta_{k}$. As in [2], since every $f \in \Omega_{k}$ satisfies the inequality $\left|\left\langle f, e_{1}\right\rangle\right| \leq\left|\left\langle f, e_{2}\right\rangle\right|$, we get that $e_{1} \in \beta_{k}(M)$. Since $\left( \pm e_{1} \pm e_{2}\right) / \sqrt{2} \in \Omega_{k}$, we have

$$
\operatorname{dist}_{k}\left(\beta_{k}(M), M\right) \geq \frac{1}{\sqrt{2}} .
$$

The same argument as in [2], p.133, can be used to show that $\beta_{k}\left(M_{k}\right)=M_{k}$. Now $k$ can be chosen large enough so that

$$
\operatorname{dist}_{e}\left(M_{k}, M\right)<\frac{1}{\sqrt{2}(n+1)},
$$

where dist $_{e}$ denotes the euclidean Hausdorff distance. Since $D \subset B_{k}$, this means that $\operatorname{dist}_{k}(G, H) \leq \operatorname{dist}_{e}(G, H)$ for every pair of closed, convex sets $G, H$. Consequently, $\operatorname{dist}_{k}\left(M_{k}, M\right)<(\sqrt{2}(n+1))^{-1}$. This gives

$$
\begin{aligned}
\operatorname{dist}_{k}\left(\beta_{k}\left(M_{k}\right), \beta_{k}(M)\right) & =\operatorname{dist}_{k}\left(M_{k}, \beta_{k}(M)\right) \\
& \geq \operatorname{dist}_{k}\left(M, \beta_{k}(M)\right)-\operatorname{dist}_{k}\left(M, M_{k}\right) \\
& >\frac{1}{\sqrt{2}}-\frac{1}{\sqrt{2}(n+1)}=\frac{n}{\sqrt{2}(n+1)} \\
& >n \operatorname{dist}_{k}\left(M, M_{k}\right)>0,
\end{aligned}
$$

which proves the assertion.

Proposition 4.3. Polyhedral norms need not have continuous ball hull mappings.

Proof. For every $n \in \mathbb{N}$, choose a natural number $k_{n}$ satisfying (15) and define $X_{n}=$ $\left(\mathbb{R}^{3},\|\cdot\|_{k_{n}}\right)$, where $\|\cdot\|_{k_{n}}$ is the polyhedral norm used in the proof of Proposition 4.2. Recall that $\|\cdot\|_{k_{n}}$ has a boundary $H_{k_{n}}$ and its ball hull mapping $\beta_{k_{n}}$ has minimal Lipschitz 
constant greater than $n$. Denote by

$$
X=\left[\bigoplus_{n \in \mathbb{N}} X_{n}\right]_{c_{0}}
$$

the $c_{0}$-sum of the family $\left\{X_{n}, n \in \mathbb{N}\right\}$, and by $\mathcal{M}, \mathcal{M}_{n}$, the families of all intersections of closed balls in $X$ and $X_{n}$, respectively. Denoting by $H_{k_{n}}$ also the image of $H_{k_{n}}$ under the canonical embedding

$$
H_{k_{n}} \subset X_{n}^{*} \hookrightarrow X^{*}=\left[\bigoplus_{n \in \mathbb{N}} X_{n}^{*}\right]_{\ell_{1}},
$$

carrying $H_{k_{n}}$ to the dual unit sphere $S_{X^{*}}$, we see that $H=\bigcup_{n} H_{k_{n}}$ is a boundary for $X$. The space $X$ is polyhedral. Indeed, let $Y \subset X$ be a finite dimensional subspace and consider $H_{Y}=\left\{f \in H: \sup f\left(B_{Y}\right)=1\right\}$. If $H_{Y}$ is infinite, we can select an infinite sequence of norm-one functionals $\left\{f_{n}\right\}_{n}$ in $H_{Y}$ and a corresponding sequence of vectors $\left\{x_{n}\right\}$ in $B_{Y}$ satisfying $f_{n}\left(x_{n}\right)=1$. Since $B_{Y}$ is compact, there is a subsequence $\left\{x_{n_{i}}\right\}$ of $\left\{x_{n}\right\}$ such that $\left\{x_{n_{i}}\right\} \rightarrow x_{0} \in B_{Y}$. Then $\lim _{i} f_{n_{i}}\left(x_{0}\right)=1$, implying that $x_{0} \notin X$, a contradiction. Therefore, $H_{Y}$ is finite and hence $B_{Y}$ is a polytope.

We may assume that, for every $n \in \mathbb{N}$ and every functional $f \in H_{k_{n}}$, the set $f^{-1}(1) \cap$ $B_{X_{k_{n}}}$ is a facet of $B_{X_{k_{n}}}$ (that is, has nonempty relative interior in $f^{-1}(1)$ ). This implies that $f^{-1}(1) \cap B_{X}$ is also a facet of $B_{X}$. Applying now Lemma 2.1 we get that $f$ is a semidenting point of $B_{X^{*}}$ and therefore $H$ turns out to be a 1-norming family of semi-denting points. We can use the representation stated in (6) for the elements of $\mathcal{M}$ to obtain that $C \in \mathcal{M}$ if and only if $C=\pi_{1}(C) \oplus \cdots \oplus \pi_{n}(C) \oplus \cdots$ and $\pi_{n}(C) \in \mathcal{M}_{n}$, for every $n \in \mathbb{N}$, where $\pi_{n}: X \rightarrow X_{n}$ denotes the canonical projection. Using again a notation of direct sums we can write

$$
\mathcal{M}=\left(\mathcal{M}_{1} \oplus \cdots \oplus \mathcal{M}_{n} \oplus \cdots\right) \cap X
$$

Let us see that the ball hull mapping associated with $\mathcal{M}$ is not continuous. To this end, consider the segments $M, M_{k_{n}}$ defined in the proof of Proposition 4.2 and define $P \subset X$ such that $\pi_{n}(P)=M$, namely $P=M \oplus \cdots \oplus M \oplus \cdots$. Then $P$ is closed, bounded, convex, and $P \notin \mathcal{M}$. Actually, $\pi_{n}\left(\beta_{X}(P)\right)=\beta_{\mathbb{R}^{3}}(M) \neq M$ for every $n \in \mathbb{N}$. Now we will construct a sequence $\left\{P_{m}\right\}_{m}$ in $\mathcal{M}$ such that $\pi_{n}\left(P_{m}\right)=M_{k_{n+m-1}}$. The idea behind the construction is to define $P_{1}=M_{k_{1}} \oplus \cdots \oplus M_{k_{n}} \oplus \cdots$ and then to apply a kind of "reverse shift operator" to obtain $P_{2}=M_{k_{2}} \oplus \cdots \oplus M_{k_{n+1}} \oplus \cdots$ and so on. Then $\operatorname{dist}\left(P_{m}, P\right)=\operatorname{dist}\left(M, M_{k_{m}}\right) \rightarrow 0$ for $m \rightarrow \infty$, while

$$
\begin{aligned}
\operatorname{dist}\left(\beta_{X}\left(P_{m}\right), \beta_{X}(P)\right) & =\operatorname{dist}\left(P_{m}, \beta_{X}(P)\right) \\
& =\operatorname{dist}\left(M_{k_{m}}, \beta_{\mathbb{R}^{3}}(M)\right) \rightarrow \operatorname{dist}\left(M, \beta_{\mathbb{R}^{3}}(M)\right)>0 .
\end{aligned}
$$




\section{REFERENCES}

[1] V. G. Boltyanski, Helly's theorem for H-convex sets, Soviet Math. Doklady 17 (1) (1976), 78-81.

[2] V. G. Boltyanski, H. Martini and P. S. Soltan, Excursions into Combinatorial Geometry. Springer, Berlin 1997.

[3] D. Chen and B.-L. Lin, On B-convex and Mazur sets of Banach spaces, Bull. Pol. Acad. Sci. Math. 43 (3) (1995), 191-198.

[4] V. P. Fonf, Polyhedral Banach spaces, Math. Notes 30 (1981), 809-813.

[5] V. P. Fonf and L. Veselý, Infinite-dimensional polyhedrality, Canad. J. Math. 56 (3) (2004), $472-494$.

[6] A. S. Granero, J. P. Moreno and R. R. Phelps, Convex sets which are intersection of closed balls, Adv. Math., 183 (1) (2004), 183-208.

[7] A. S. Granero, J. P. Moreno and R. R. Phelps, Mazur sets in normed spaces, Discrete Comput. Geom. 31 (2004), 411-420.

[8] B. Grünbaum, Convex Polytopes (Prepared by Volker Kaibel, Victor Klee, and Günter M. Ziegler), 2nd ed., Graduate Texts in Mathematics 221, Springer, New York 2003.

[9] V. Klee, Polyhedral sections of convex bodies, Acta Math. 103 (1960), 243-267.

[10] J. P. Moreno, P. L. Papini and R. R. Phelps, Diametrically maximal and constant width sets in Banach spaces, Canad. J. Math., to appear.

[11] J. P. Moreno, Semicontinuous functions and convex sets in $C(K)$ spaces, preprint.

[12] R. Schneider, Convex Bodies: The Brunn-Minkowski Theory, Encyclopedia of Mathematics and its Applications, 44. Cambridge University Press, Cambridge 1993.

[13] P. Terán, Intersections of closed balls and expectations of random sets, preprint.

[14] G. M. Ziegler, Lectures on Polytopes, Graduate Texts in Mathematics, 152. Springer, New York 1995.

JOSÉ PEDRO MOREnO: Dpto. Matemáticas, Facultad de Ciencias, Universidad Autónoma DE MADRID, MADRID 28049, SPAIN. josepedro.moreno@uam.es

ROLF SCHNEIDER: Mathematisches Institut, Albert-Ludwigs-Universität, D-79104 FREIBURG I. BR., GERMANY. rolf.schneider@math.uni-freiburg.de 